\title{
Fractal geometry for animal biometrics: a response to Kühl and Burghardt
}

\author{
Roger Jovani ${ }^{1}$, Lorenzo Pérez-Rodríguez ${ }^{2,3}$, and François Mougeot ${ }^{4,5}$ \\ ${ }^{1}$ Department of Evolutionary Ecology, Estación Biológica de Doñana, CSIC, Avda. Americo Vespucio, s/n, 41092, Seville, Spain \\ ${ }^{2}$ CIBIO, Centro de Investigação em Biodiversidade e Recursos Genéticos, Campus Agrário de Vairão, Rua Padre Armando Quintas, \\ 4485-661 Vairão, Portugal \\ ${ }^{3}$ Department of Evolutionary Ecology, Museo Nacional de Ciencias Naturales (MNCN-CSIC), José Gutiérrez Abascal 2, 28006, \\ Madrid, Spain \\ ${ }^{4}$ Instituto de Investigación en Recursos Cinegéticos, IREC (CSIC-UCLM-JCCM), Ronda de Toledo, s/n, 13005, Ciudad Real, Spain \\ ${ }^{5}$ Estación Experimental de Zonas Áridas (EEZA-CSIC), Ctra. de Sacramento s/n La Cañada de San Urbano, 04120, Almería, Spain
}

In their recent article in TREE, Kühl and Burghardt [1] reviewed the emerging field of animal biometrics, but missed an opportunity to highlight an important and promising tool: fractal geometry. This tool has seldom been applied in the context of animal biometrics, but the few tentative examples reported so far have shown great potential to contribute to the development of this research field.

Fractals are mathematical sets that are self-similar across scales [2]. Natural examples of fractal-like behaviour include patterns that are built by recursive iteration, such as branching (e.g., roots, clouds, or lungs), where a small part ends up resembling the whole. Interestingly, fractal geometry can also describe patterns that are not strictly selfsimilar, but that show intricate, complex, and heterogeneous configurations. For this reason, fractal geometry offers new and valuable opportunities to describe and compare complex individual- or species-specific patterns.

As highlighted by Kühl and Burghardt [1], animal biometric tools characterise phenotypic appearance, provide ways to recognise phenotypes, and enable the profiling and description of individual behaviours. Some pioneering studies have shown the potential of fractal geometry for such tasks, by measuring the complexity of natural patterns through their fractal dimension (FD). For instance, FD can discriminate between butterfly species from their wing patterns [3], branchiopod morph types from the structure of their eggshells [4], mammalian species from their cranial suture patterns [5], and ammonoid taxa from their shell suture patterns [6]. Fractal analysis can also differentiate types of music and instruments [7], suggesting a potential use for the study of animal acoustic signals. Individual and collective animal behaviours have also been successfully characterised by fractal geometry. Examples include analyses of movement tracks [8], spatial distribution of individuals or nests [9,10], or burrow architecture of subterranean species [11]. Finally, black-and-white plumage patterns have also been described using fractal geometry, with variation in the FD pattern being shown experimentally to relate to individual quality [12].

As for most other tools used in animal biometrics, images of patterns require prior processing and standardising before fractal geometry analysis. Also, understanding what

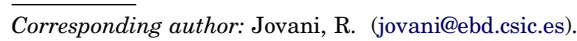

variation in FD means for a particular pattern (i.e., which pattern properties cause variation in FD) may not be as straightforward as with tools designed to measure a particular feature of a pattern. However, the latter can turn into an advantage because understanding the meaning of FD for a particular pattern may unravel unknown features of the pattern under study. The main limitation may be that a similar FD for a given structure could be shared by different individuals or species. When that is the case, fractal dimension alone would be a bad choice, but could be used as one more trait for pattern matching or as an initial filter to discard improbable matches.

The advantages and opportunities that fractal geometry offers to animal biometric studies are several. First, measuring the FD of an object or pattern is relatively straightforward, and several methods (e.g., box-counting, dividers method [2,5,12]), and software tools are readily available. Second, fractal geometry captures the scaling properties of the object, and is scale invariant, something that is particularly useful when studying objects of different size, or comparing images taken at different distances. Third, the FD of an object or pattern provides a single value that summarises the way the pattern 'behaves' across scales. This could have biological relevance, because it mirrors the way individuals develop signalling traits in a coherent way across scales (e.g., from individual feathers to whole plumages for bird patterns), which could convey information (e.g., physiological state) about the individual displaying the pattern [12]. Fourth, it can be applied to a wide array of patterns, and software tools developed for the analysis of a given pattern in a given species could easily be adapted to the study of other patterns.

For all these reasons, we suggest that the field of animal biometrics would benefit greatly from incorporating fractal geometry analyses into the study of animal patterns, and we hope that this letter will stimulate further uses and applications within this research field.

\section{Acknowledgements}

R.J. is supported by a 'Ramón y Cajal' (RYC-2009-03967) program from the Spanish Ministerio de Ciencia e Innovación (MICINN). L.P-R. is supported by a postdoctoral contract from ICETA-CCDRN.

\section{References}

1 Kühl, H.S. and Burghardt, T. (2013) Animal biometrics: quantifying and detecting phenotypic appearance. Trends Ecol. Evol. 28, 432-441 
2 Mandelbrot, B.B. (1983) The Fractal Geometry of Nature. W.H. Freeman

3 Castrejón-Pita, A.A. et al. (2004) Fractal dimension in butterflies wings: a novel approach to understanding wing patterns? J. Math. Biol. 50, 584-594

4 Bruner, E. et al. (2013) Fractal analysis of the egg shell ornamentation in anostracans cysts: a quantitative approach to the morphological variations in Chirocephalus ruffoi. Hydrobiologia 705, 1-8

5 Gibert, J. and Palmqvist, P. (1995) Fractal analysis of the Orce skull sutures. J. Hum. Evol. 28, 561-575

6 Pérez-Claros, J.A. et al. (2002) First and second orders of suture complexity in ammonites: a new methodological approach using fractal analysis. Math. Geol. 34, 323-343

7 Hsu, K.J. (1993) Fractal geometry of music: from bird songs to Bach. In Applications of Fractals and Chaos (Crilly, A. et al., eds), pp. 21-39, Springer-Verlag
8 Dicke, M. and Burrough, P.A. (1988) Using fractal dimension for characterising tortuosity of animal trails. Physiol. Entomol. 13, 393-398

9 Jovani, R. and Tella, J.L. (2007) Fractal bird nest distribution produces scale-free colony sizes. Proc. R. Soc. B 274, 2465-2469

10 Lander, M.E. et al. (2011) Spatial patterns and scaling behaviors of Steller sea lion (Eumetopias jubatus) distributions and their environment. J. Theor. Biol. 274, 74-83

11 Le Comber, S.C. et al. (2006) Burrow fractal dimension and foraging success in subterranean rodents: a simulation. Behav. Ecol. 17, 188195

12 Pérez-Rodríguez, L. et al. (2013) Fractal geometry of a complex plumage trait reveals bird's quality. Proc. $R$. Soc. B 280, 20122783 http://dx.doi.org/10.1098/rspb.2012.2783

0169-5347/\$ - see front matter @ 2013 Elsevier Ltd. All rights reserved. http://dx.doi.org/10.1016/j.tree.2013.06.004 Trends in Ecology \& Evolution xx (2013) 1-2 